\title{
Second-order schedules: Manipulation of brief-stimulus duration at component completion*
}

\author{
STEVEN L. COHEN, JOAN E. HUGHES, and D. ALAN STUBBS \\ University of Maine, Orono, Maine 04473
}

\begin{abstract}
In a second-order schedule, fixed-interval components were reinforced according to a variable-interval schedule. A brief stimulus accompanied the completion of each fixed interval. Brief-stimulus duration was varied across conditions from 0.5 to $8 \mathrm{sec}$. Patterning was greater the longer the duration of the stimulus. Additionally, exposure to relatively long brief-stimulus durations enhanced patterning upon reexposure to shorter brief-stimulus durations.
\end{abstract}

Contemporary research in the area of conditioned reinforcement has made extensive use of second-order schedules of reinforcement. Kelleher has defined a second-order schedule as a schedule which "... treats a pattern of behavior engendered by a schedule contingency as a unitary response that is itself reinforced according to some schedule of reinforcement [1966, p. 476]." In numerous studies when the completion of schedule components resulted in the brief presentation of a stimulus, rates and patterns of responding similar to rates and patterns of responding engendered by presentations of primary reinforcers have been observed (e.g., Kelleher, 1966; Marr, 1969). Recent research (Stubbs, 1971) has shown that the properties of brief stimuli play an important role in determining the degree of patterns of responding. Different types of stimuli (e.g., keylight or blackout) used in brief-stimulus procedures and the number of stimuli involved in the stimulus change may influence performance. Another aspect of brief stimuli which may affect behavior is that of duration. Although duration of the brief stimulus is a feature in all brief-stimulus procedures, the effect of duration of the brief stimulus has been reported in only a few studies to date (Neuringer \& Chung, 1967; Staddon \& Innis, 1969). The purpose of the present study was to systematically assess the changes in performance which result from manipulations of brief-stimulus duration under a second-order schedule.

\section{METHOD}

\section{Subjects and Apparatus}

Two adult male White Carneaux pigeons were maintained at $80 \%$ of their free-feeding body weights. Both pigeons had extensive experimental histories at the start of the experiment

*The first author held a National Aeronautics and Space Administration traineeship. The second author held an NDEA Title IV fellowship. Reprints may be obtained from the authors. Department of Psychology, University of Maine, Orono, Maine 04473. (see Stubbs \& Cohen, 1972). The experimental space was a standard two-key pigeon chamber housed in a room with white masking noise continuously present. Standard relay and timing circuitry, located in an adjacent room, controlled experimental sessions.

\section{Procedure}

The pigeons responded under a second-order schedule in which behavior controlled by fixed-interval (FI) components was maintained according to a variable-interval (VI) schedule of reinforcement. The value of the FI schedule was $48 \mathrm{sec}$. The VI schedule had an average interval of $240 \mathrm{sec}$ and contained 16 intervals (Catania \& Reynolds formulation, 1968, pp. 380-381). The right key was transilluminated red; the left key was dark and ineffective. If a time period of the VI schedule had not elapsed, the completion of an FI 48-sec component produced a change in the keylight illumination from red to green. If a time period of the VI schedule had elapsed, the completion of an FI 48-sec component produced 4-sec access to grain. A preceding, nonoverlapping pairing of the brief-stimulus presentation and grain reinforcement was used under all conditions. The completion of an FI 48-sec component scheduled to produce food initiated the onset of the brief stimulus. At the termination of the brief stimulus, the key was darkened and the 4-sec food cycle was initiated. This schedule may be designated as VI $240 \mathrm{sec}$ (FI $48 \mathrm{sec}: \mathrm{S}^{\mathrm{p}}$ ) (Stubbs, 1971). The food hopper was illuminated with blue light. Each session was terminated following 26 food presentations.

The duration of the brief-stimulus presentation at the completion of each FI 48-sec component was manipulated over the course of the study. Three duration values were used: $0.5,2$, and $8 \mathrm{sec}$. Table 1 shows the order of presentation of each experimental condition and the number of sessions under each condition for both pigeons. The pigeons were exposed to a particular condition until no systematic changes in behavior were observed for at least five sessions.

\section{RESULTS}

Figure 1 shows representative cumulative records under each of the six experimental conditions for Pigeon 5. The records at the left of Fig. 1 were taken from Conditions 1, 2, and 3 (first determination conditions). The records on the right of Fig. 1 were taken from Conditions 4,5 , and 6 (redetermination conditions). 
Table 1

Conditions in Order of Presentation and the Number of Sescions Under Each*

\begin{tabular}{ccc}
\hline $\begin{array}{c}\text { Duration of the } \\
\text { Brief Stimulus }\left(\mathbf{S}^{\mathbf{P}}\right)\end{array}$ & \multicolumn{2}{c}{ Number of Sessions } \\
\cline { 2 - 3 } (in sec) & Pigeon 5 & Pigeon 15 \\
\hline 0.5 & 34 & 31 \\
2.0 & 23 & 17 \\
8.0 & 19 & 16 \\
0.5 & 20 & 19 \\
2.0 & 12 & 10 \\
8.0 & 7 & 7 \\
\hline
\end{tabular}

The schedule across conditions was VI 240-sec (FI 48-sec: $\boldsymbol{S P}^{P}$.

All records show patterns of responding within components appropriate to Fl schedules. At all durations, the pattern of responding within components was typified by pausing followed by positively accelerated rates of responding. However, at the $0.5-\mathrm{sec}$ duration, high rates of responding immediately or shortly following some stimulus presentations did occur.
When the duration of the brief-stimulus presentation was increased to $2 \mathrm{sec}$. fewer instances of high response rates immediately following the brief-stimulus presentation occurred. At the 8-sec duration, pausing occurred after each stimulus presentation and lasted for a longer period before responding resumed. Overall response rates tended to be slightly lower as durations were increased, due in large part to longer and more consistent pausing after brief-stimulus presentations.

At duration values of 0.5 and $2.0 \mathrm{sec}$. performance during redetermination conditions showed a consistent enhancement in patterning as compared to performance during original exposure conditions; fewer instances of high response rates immediately following brief-stimulus presentations occurred, and longer pauses were observed.

The performance of Pigeon 15 was quite similar, although this pigeon responded at a higher response rate over all experimental conditions.

Figure 2 shows index of curvature measures as a function of brief-stimulus duration for both pigeons. The index of curvature is a statistic which describes the extent of patterning under FI schedules (Fry, Kelleher,

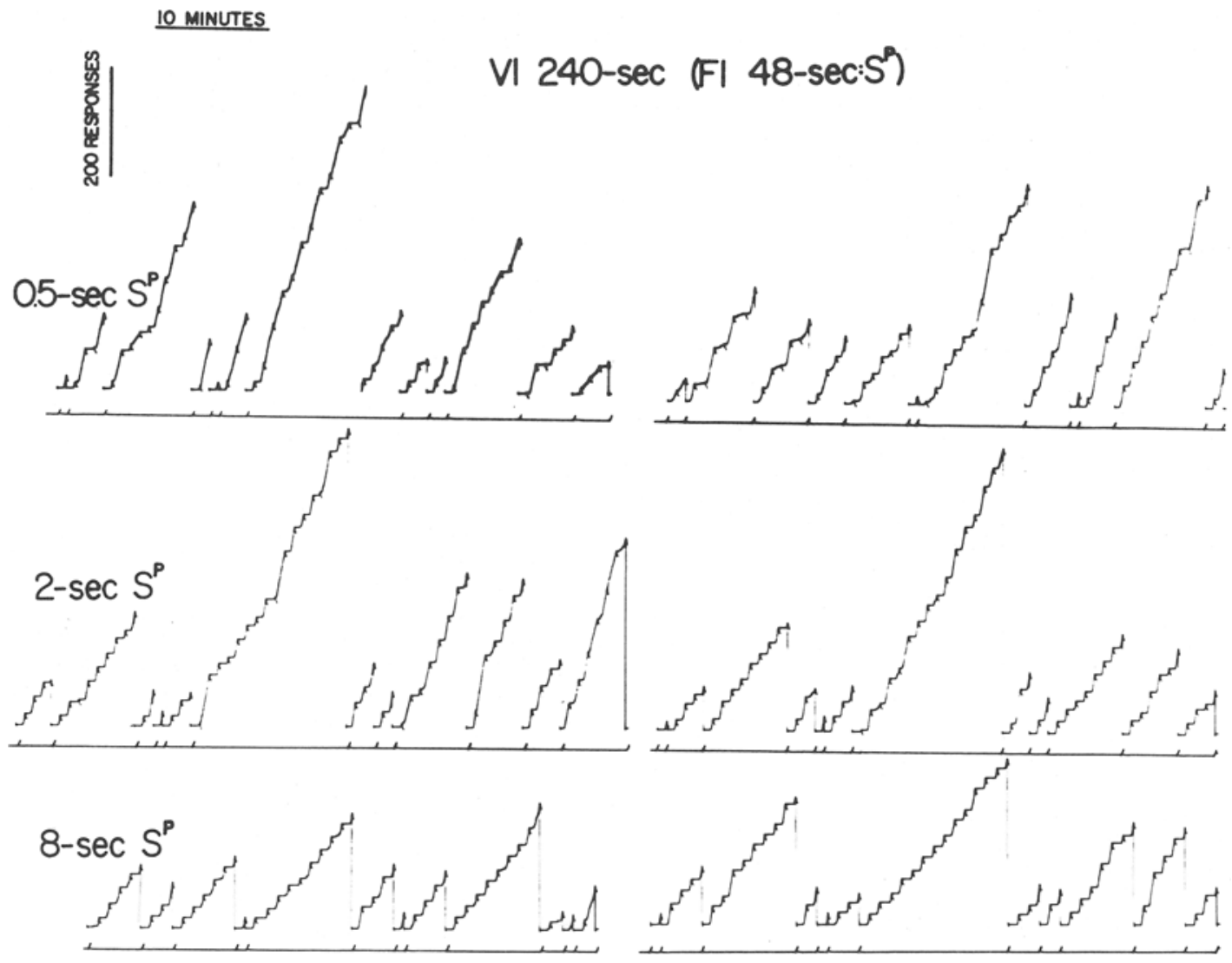

Fig. 1. Portions of cumulative response records taken from the last session of each condition. Records on the left are from original determinations at each stimulus duration. Records on the right are from redeterminations at each stimulus duration. The label $\mathbf{s}^{p}$ indicates that the brief stimulus was paired with food. Each response stepped the pen once. Brief-stimulus presentations are represented by the deflection of the response pen. Food presentations are indicated by deflections of event pen and the resetting of the response pen. 
\& Cook, 1960). Higher values (reaching a maximum of 0.75 when the fixed interval is divided into quarters) indicate greater curvature.

In general, index measures increased with increasing brief-stimulus duration; the functions are negatively accelerated and appear to reach an asymptote at approximately 2 sec. Additionally, greater response patterning was observed under redetermination conditions at the 0.5 - and $2-\mathrm{sec}$ durations. The elevated functions for redetermination conditions suggest a trend toward increased patterns of responding at short brief-stimulus durations following exposure to longer durations. This result was particularly evident for Pigeon 5. Comparisons of the ranges of index measures over the last five sessions of each condition for Pigeon 5 show no overlap of index measures between first and second determinations at both 0.5 - and $2-\mathrm{sec}$ durations. For Pigeon 15, although median index measures were higher under redetermination conditions at both 0.5 . and 2-sec durations, overlap in the ranges did occur. It might be argued that the enhancement of patterning seen from first to second determinations was the result of a trend toward greater patterning simply as a function of the number of sessions in effect. However, inspection of the data from original exposures does not support this interpretation. The median index measures over four successive 5-day blocks of the last 20 sessions of the $0.5-\mathrm{sec}$ experimental condition (first determination) were: $0.37,0.31,0.40$, and 0.38 for Pigeon 5 and 0.33 , $0.42,0.44$, and 0.44 for Pigeon 15. Thus, the degree of patterning was stable for $15-20$ sessions prior to the change from the first to the second condition.

\section{DISCUSSION}

The data reveal two major findings. First, performance was affected by the duration of the brief stimulus: patterning increased as a function of increases in stimulus duration. Second, the data suggest that exposure to relatively long brief-stimulus durations may enhance patterning upon reexposure to shorter durations.

Two factors might have contributed to the brief-stimulus effects on patterning. First, the brief stimulus was intermittently paired with food. This pairing operation has been viewed as an important operation for establishing the stimulus as a conditioned reinforcer (Kelleher, 1966). The patterning within components could have been produced by the presentation of a conditioned reinforcer at the completion of each component. A second factor is the discriminative effects of the brief stimulus (Stubbs, 1971). When, for example, a second-order schedule contains FI components as in the present case, there is a fixed temporal relation between the presentation of the brief stimulus and the presentation of food. Accordingly, the brief stimulus is always followed by a period of nonreinforcement. The period of

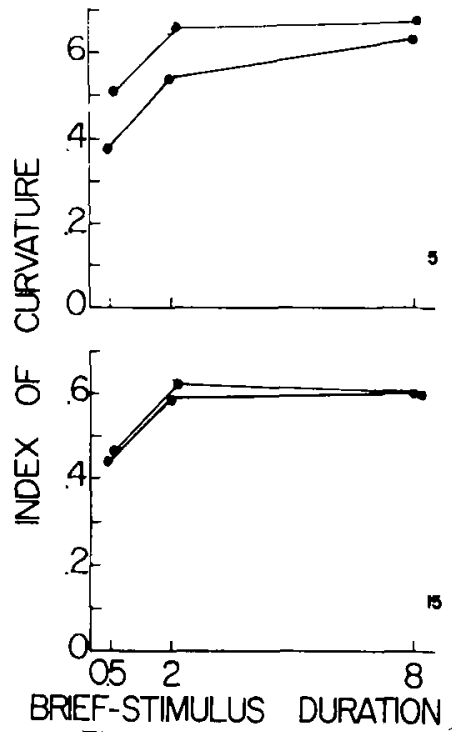

Fig. 2. Index of curvature measures as a function of brief-stimulus duration. Filled circles represent medians of the last five sessions at each duration value. Where two points are shown at each duration value, points displaced slightly to the left are from first determination conditions.

nonreinforcement following the stimulus presentations could serve to lower response rates following each brief stimulus. The intermittent presentation of food, only terminating some components, would control the positively accelerated rates. The degree of response patterning may be a function in part of the degree of discriminative control exerted by the brief stimulus with respect to periods of nonreinforcement.

The degree of stimulus control may in turn depend on the saliency of the brief stimulus. Recent evidence (Stubbs, 1971; Stubbs \& Cohen, 1972) has shown that patterning is affected by different properties of the brief stimulus such as the number of stimuli involved (e.g., keylight vs keylight plus houselight) and the type of stimulus (e.g.. keylight vs blackout). The present data indicate that the duration of a stimulus also is a property that affects patterning. Increases in the number of stimuli involved, increases in the duration of a stimulus, and changes in the type of stimulus all may make a stimulus more salient and increase its discriminative control over responding.

The effects of brief-stimulus duration on patterning are consistent with data reported by Staddon and Innis (1969). Staddon and Innis used a reinforcement-omission procedure in which animals responded on a FI schedule and blackouts were intermittently substituted for food presentations. This schedule can be considered a second-order schedule in which blackouts served as a brief stimulus presented at component completion. With pigeons, Staddon and Innis found increased pausing following a blackout as the duration of the blackout increased from 2 to $32 \mathrm{sec}$. The increased pausing suggests greater $F I$ patterning as duration increased. The present data and the data of Staddon and Innis seemingly differ from those of 
Neuringer and Chung (1967). Neuringer and Chung varied the duration of a brief stimulus $(0.25,1.0$. and $7.0 \mathrm{sec}$ ) in a second-order schedule in which response-initiated FI components were reinforced according to a VI schedule. No systematic rate differences occurred as a function of brief-stimulus duration. There are numerous procedural differences between the present study and that of Neuringer and Chung (e.g., different schedule parameters, different reinforcement rates, different brief stimuli). However, the different findings might be more a function of different analysis than of procedures. Neuringer and Chung did not examine the effects of brief-stimulus duration on patterning; such an analysis might have shown differences as a function of brief-stimulus duration. In the present study, response rates were similar across conditions, with a trend toward lower rates at longer durations of the brief stimulus.

The second major finding of the present study was that exposure to long brief-stimulus durations enhanced patterning under second-order schedules when conditions were returned to shorter duration values, suggesting a partial degree of irreversibility in the established stimulus control. The lack of reversibility is not unique to the present experiment and has been found in other situations (e.g., the "metastability" phenomenon of Staddon, 1965). If the effects of exposure to long brief-stimulus durations show a degree of irreversibility, similar results might occur with other changes in stimulus properties. Of special interest are operations involving the pairing and nonpairing of the stimulus with primary reinforcers. In some second-order schedule experiments, one brief stimulus was used as a paired stimulus while a different stimulus was used as a nonpaired stimulus. Stubbs (1971) has pointed out that differences between pairing and nonpairing conditions are confounded by stimulus differences and suggested that the same stimulus be used for both the pairing and nonpairing operations. When, however, the same stimulus has been used under both paired and nonpaired conditions, the following results have been observed with some pigeons (but by no means all or even a majority): patterning stabilized at one level under the initial experimental condition when the stimulus was nonpaired; the degree of patterning increased somewhat when the stimulus was paired and remained at the same level when the nonpairing operation was reinstated
(Stubbs \& Cohen, 1972). Possibly the pairing operation might have. for some pigeons, produced some irreversible effects on patterning. ${ }^{1}$ Stated more generally. once a high level of patterning has been established. the degree of patterning might tend to remain high in spite of changes in the properties of the brief stimulus.

\section{REFERENCES}

Catania. A. C., \& Reynolds, G. S. A quantitative analysis of the responding maintained by interval schedules of reinforcement. Journal of the Experimental Analysis of Behavior, 1968, 11 , 327-383.

Fry, W., Kelleher, R. T., \& Cook, L. A mathematical index of performance on fixed-interval schedules of reinforcement. Journal of the Experimental Analysis of Behavior, 1960, 3 , 193-199.

Kelleher, R. T. Conditioned reinforcement in second-order schedules. Journal of the Experimental Analysis of Behavior, $1966,9,475-485$.

Marr, M. J. Second-order schedules. In D. P. Hendry (Ed.), Conditioned reinforcement. Homewood, IL: Dorsey Press, 1969. Pp. 37-60.

Neuringer, A. J., \& Chung, S.-H. Quasi-reinforcement: Control of responding by a percentage-reinforcement schedule. Journal of the Experimental Analysis of Behavior, 1967, 10, 45-54.

Staddon, J. E. R. Some properties of spaced responding in pigeons. Journal of the Experimental Analysis of Behavior, $1965,8,19-27$

Staddon, J. E. R., \& Innis, N. K. Reinforcement omission on fixed-interval schedules. Journal of the Experimental Analysis of Behavior, 1969, 12, 689-700.

Stubbs, D. A. Second-order schedules and the problem of conditioned reinforcement. Journal of the Experimental Analysis of Behavior, 1971, 16, 289-313.

Stubbs, D. A., \& Cohen, S. L. Second-order schedules: Comparison of different procedures for scheduling paired and nonpaired brief stimuli. Journal of the Experimental A nalysis of Behavior, 1972, 18, 403-413.

\section{NOTE}

1. The increase in patterning with some pigeons might have little to do with the introduction of the pairing procedure. Stubbs (1971) has noted that for many pigeons, patterning changed from the first condition to the second regardless of the specific change (e.g., change from nonpairing to pairing operation, change from pairing to nonpairing operation, changes in schedule parameters, etc.).

(Received for publication January 3, 1973; accepted January 5,1973 .) 